\title{
The topological uniqueness of the deltahedra found in the boranes $\mathrm{B}_{n} \mathrm{H}_{n}^{2-}(6 \leqslant n \leqslant 12)$
}

\author{
R. B. King \\ Department of Chemistry, University of Georgia, Athens, GA 30602 (U.S.A.) \\ and A. J. W. Duijvestijn \\ Department of Informatics, University of Twente, 7500 AE Enschede (The Netherlands)
}

(Received July 16, 1990)

\begin{abstract}
The deltahedra observed experimentally in the borane anions $\mathrm{B}_{n} \mathrm{H}_{n}{ }^{2-}(6 \leqslant n \leqslant 10)$ are the only possible $n$-vertex deltahedra having only degree 4 and 5 vertices. The existence of an 11-vertex deltahedron having only degree 4 or 5 vertices is topologically impossible in accord with the presence of a degree 6 vertex in the observed structure for $\mathrm{B}_{11} \mathrm{H}_{11}{ }^{2-}$.
\end{abstract}

The deltahedral boranes, $\mathrm{B}_{n} \mathrm{H}_{n}{ }^{2-}$, and the isoelectronic carboranes, $\mathrm{C}_{2} \mathrm{~B}_{n-2} \mathrm{H}_{m}$, are the prototypical examples of globally delocalized molecules exhibiting three-dimensional aromaticity [1-6]. A characteristic feature of the structure of such molecules is the tendency for the boron and carbon atoms to form deltahedra in which all vertices have degrees 4 or 5. (In the context of this discussion deltahedra are polyhedra in which all faces are triangles and the degree of a vertex is the number of edges meeting at that vertex.) The deltahedra found in the boranes $\mathrm{B}_{n} \mathrm{H}_{n}{ }^{2-}$ for $n-6,7,8,9,10$ and 12 are depicted in Fig. $1[7,8]$. All of these deltahedra contain only degree 4 and degree 5 vertices. However, the 11 vertex deltahedron found in $\mathrm{B}_{11} \mathrm{H}_{11}{ }^{2-}$ [9] is the socalled edge-coalesced icosahedron (Fig. 2), which contains a single vertex of degree 6 as well as eight vertices of degree 5 and two vertices of degree 4 .

The shapes of the boron deltahcdra in the boranes $\mathrm{B}_{n} \mathrm{H}_{n}{ }^{2-}(6 \leqslant n \leqslant 12)$ raise the following topological questions.

(i) Are the deltahedra found in the structures with $6,7,8,9,10$ and 12 boron vertices the only possible deltahedra in which all vertices have degrees 4 or 5 ?

(ii) Is there a deltahedron with 11 vertices in which all of the vertices have degrees 4 or 5 ?

This note explores briefly these two questions.

In connection with the first of these questions, previously reported methods $[10,11]$ were used to generate all three-connected planar graphs having

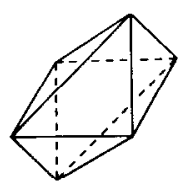

Octahedron

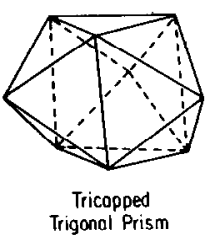

Fig. 1. The deltahedra found in the boranes $\mathrm{B}_{n} \mathrm{H}_{n}{ }^{2-}(n=6$, $7,8,9,10$ and 12 ) in which all vertices have degrees 4 or 5 .

only degree 4 and 5 vertices and 9 or less total vertices and having 10 total vertices and 23 or 24 edges. All such graphs correspond to polyhedra. The numbers of topologically distinct polyhedra so obtained having only degree 4 and 5 vertices, 6 to 11 total vertices, and up to 24 total edges are given in Table 1; further details on specific polyhedra enumerated in Table 1 are published elsewhere [12]. Of particular chemical significance is the existence of only one topologically distinct deltahedron with only degree 4 and 5 vertices for each number of total vertices from 6 to 10 , inclusive. These deltahedra, 
TABLE 1 . Numbers of topologically distinct polyhedra containing only vertices of degrees 4 and $5^{\mathrm{a}}$

\begin{tabular}{|c|c|c|c|c|c|c|c|c|c|c|c|c|c|}
\hline & \multicolumn{13}{|c|}{ Number of edges } \\
\hline & 12 & 13 & 14 & 15 & 16 & 17 & 18 & 19 & 20 & 21 & 22 & 23 & 24 \\
\hline Total & 1 & 0 & 0 & 1 & 1 & 1 & 2 & 3 & 5 & 9 & 14 & $b$ & b \\
\hline 6 vertices & $1 \mathrm{D}$ & 0 & 0 & 0 & 0 & 0 & 0 & 0 & 0 & 0 & 0 & 0 & 0 \\
\hline 7 vertices & 0 & 0 & 0 & $1 \mathrm{D}$ & 0 & 0 & 0 & 0 & 0 & 0 & 0 & 0 & 0 \\
\hline 8 vertices & 0 & 0 & 0 & 0 & 1 & 1 & $1 \mathrm{D}$ & 0 & 0 & 0 & 0 & 0 & 0 \\
\hline 9 vertices & 0 & 0 & 0 & 0 & 0 & 0 & 1 & 3 & 2 & $1 \mathrm{D}$ & 0 & 0 & 0 \\
\hline 10 vertices & 0 & 0 & 0 & 0 & 0 & 0 & 0 & 0 & 3 & 8 & 11 & 2 & 1D \\
\hline 11 vertices & 0 & 0 & 0 & 0 & 0 & 0 & 0 & 0 & 0 & 0 & 3 & $b$ & $b$ \\
\hline
\end{tabular}

${ }^{a}$ The deltahedra are indicated by D. ${ }^{b}$ The numbers of polyhedra with 23 and 24 edges, 11 or more vertices, and only vertices of degrees 4 and 5 were not determined because of the complexity of the problem and limited direct chemical relevance since they clearly are not deltahedra.

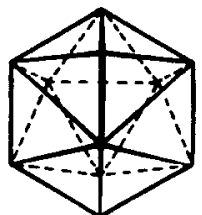

Fig. 2. The edge-coalesced icosahedron found in $\mathrm{B}_{11} \mathrm{H}_{11}^{2-}$. Note that this deltahedron contains a single degree 6 vertex in addition to eight vertices of degree 5 and two vertices of degree 4.

of course, correspond to those found experimentally $[7,8]$ in $\mathrm{B}_{n} \mathrm{H}_{n}{ }^{2-}(6 \leqslant n \leqslant 10)$.

The second question concerns the existence of a deltahedron with 11 total vertices, all of degree 4 or 5 . Such an 11-vertex deltahedron necessarily must have 27 edges, 18 faces, 10 vertices of degree 5, and 1 vertex of degree 4 on the basis of Euler's theorem and other elementary topological considerations [13]. The duals [14] of deltahedra are the simple polyhedra, namely polyhedra with all vertices of degree 3 . Simple polyhedra must satisfy the relationship [15]

$3 f_{3}+2 f_{4}+f_{5}=12+\sum_{k \geqslant 7}(k-6) f_{k}$

in which $f_{k}$ is the number of faces having $k$ edges (i.e. $f_{3}$ is the number of triangular faces, $f_{4}$ the number of quadrilateral faces, etc.). In the dual of the 11-vertex deltahedron of interest $f_{3}=f_{\mathrm{k}}(k \geqslant 7)=0$ so that $f_{4}=1$ and $f_{5}=10$ from eqn. (1). Equation (1) says nothing about the required value of $f_{6}$, i.e. the number of hexagonal faces corresponding to the number of degree 6 vertices in the dual polyhedron. However, the corresponding minimum values of $f_{6}$ have been determined by hand [15-17] for all sets of $f_{3}, f_{4}$ and $f_{5}$ satisfying eqn. (1) for $f_{\mathrm{k}}=0$ for $k \geqslant 7$. In the case of $f_{3}=0, f_{4}=1, f_{5}=10$ corresponding to the dual of the 11-vertex deltahedron of interest, the minimum value of $f_{6}$ is 2 rather than 0 . This indicates the topological impossibility of a simple polyhedron with a total of 11 faces, all of which are quadrilaterals or pentagons or of the dual of such a simple polyhedron, namely a deltahedron with 11 total vertices, all of degree 4 or 5 .

The observations outlined above suggest that the $\mathrm{B}_{n} \mathrm{H}_{n}{ }^{2-}$ deltahedra are uniquely defined by simple topological considerations with vertices of degrees 4 and 5 being strongly favored. Thus the deltahedra found in $\mathrm{B}_{n} \mathrm{H}_{n}{ }^{2-}(6 \leqslant n \leqslant 10)$ are the only possible deltahedra having only degree 4 and 5 vertices and $n$ total vertices. Only in the case of $\mathrm{B}_{11} \mathrm{H}_{11}{ }^{2-}$ where an 11-vertex deltahedron having only degree 4 and 5 vertices is shown to be topologically impossible, does the observed deltahedron contain a single degree 6 vertex.

\section{References}

1 J. Aihara, J. Am. Chem. Soc., 100 (1978) 3339.

2 R. B. King and D. H. Rouvray, J. Am. Chem. Soc., 99 (1977) 7834.

3 R. B. King, in R. B. King (ed.), Chemical Applications of Topology and Graph Theory, Elsevier, Amsterdam, 1983, pp. 99-123.

4 R. B. King, in J. F. Liebman and A. Greenberg (eds.), Molecular Structure and Energetics, Verlag Chemie, Deerfield Beach, FL, 1986, pp. 123-148.

5 R. B. King, J. Math. Chem., I (1987) 249.

6 R. B. King, J. Math. Chem., 4 (1990) 69.

7 E. L. Muetterties and W. H. Knoth, Polyhedral Boranes, Marcel Dekker, New York, 1968.

8 R. L. Middaugh, in E. L. Muetterties (ed.), Boron Hydride Chemistry, Academic Press, New York, 1975, pp. 273-300.

9 F. Klanberg and E. L. Muetterties, Inorg. Chem., 5 (1966) 1955.

10 A. J. W. Duijvestijn and P. J. Federico, Math. Comput., 37 (1981) 523. 
11 A. J. W. Duijvestijn, Philips Res. Rep., 17 (1962) 523.

12 A. J. W. Duijvestijn and B. Helthuis, to be published.

13 R. B. King, in M. Gielen (ed.), Advances in Dynamic Stereochemistry, Vol. 2, Freund, Tel Aviv, 1988, pp. 1-36.

14 B. Grünbaum, Convex Polytopes, Interscience, New York, 1967, pp. 46-51.
15 B. Grünbaum, Convex Polytopes, Interscience, New York, 1967, pp. 253-271.

16 V. Eberhard, Zur Morphologie der Polyeder, Leipzig, 1891 , as cited in ref. 15.

17 M. Brückner, Vielecke und Vielflache, Teubner, Leipzig, 1900 , as cited in ref. 15 . 